\title{
DOXAZOSINA EN FORMULACIÓN DE LIBERACIÓN RETARDADA EN LA RETENCIÓN AGUDA DE ORINA POR HIPERPLASIA BENIGNA DE PRÓSTATA. ACCIÓN DEL INCREMENTO DE DOSIS SOBRE EL EFECTO RESCATE
}

\author{
J.A. LORENTE GARÍN, D. CAÑIS SÁNCHEZ, O. ARANGO TORO, O. BIELSA GALI, \\ R. CORTADELLAS ÁNGEL, A. GELABERT MAS
}

Servicio y Cátedra de Urología. Hospital del Mar. Universidad Autónoma de Barcelona. Barcelona.

Actas Urol Esp. 28 (1): 32-37, 2004

\section{RESUMEN}

DOXAZOSINA EN FORMULACIÓN DE LIBERACIÓN RETARDADA EN LA RETENCIÓN AGUDA DE ORINA POR HIPERPLASIA BENIGNA DE PRÓSTATA

OBJETIVO: Estudio prospectivo y randomizado para valorar la utilidad de la doxazosina en formulación de liberación retardada en la retención aguda de orina (RAO) por hiperplasia benigna de próstata (HBP).

MATERIAL Y MÉTODOS: Se estudia una cohorte de 40 varones consecutivos con un primer episodio de RAO por HBP. Veinte son tratados aleatoriamente durante 7 días con $4 \mathrm{mg}$ de doxazosina de liberación retardada antes de la retirada del catéter. Los pacientes no tratados que no restablecieron las micciones espontáneas recibieron también $4 \mathrm{mg}$ de doxazosina. Finalmente, todos los pacientes que persistieron con catéter por retirada infructuosa fueron tratados con $8 \mathrm{mg}$ de doxazosina, evaluándose el porcentaje de pacientes respondedores. Se determina mediante análisis de regresión logistica el valor predictivo de la respuesta al tratamiento de la edad, IPSS, 马oL, volumen de orina retenido, volumen prostático y tiempo de evolución del prostatismo.

RESULTADOS: El 82,5\% de los pacientes (33/40) consiguieron la micción tras la retirada del catéter. El 84,8\% (28/33) fueron tratados con doxazosina ( 21 con $4 \mathrm{mg}$ y 7 con $8 \mathrm{mg}$ ). En el primer intento de retirada, el 60\% de los pacientes (12/20) tratados con $4 \mathrm{mg}$ de doxazosina consiguieron micción espontánea, mientras tan sólo el $25 \%(5 / 20)$ de los no tratados, p=0,02. Igualmente, el $60 \%$ de los pacientes (9/15) tratados con $4 \mathrm{mg}$ de doxazosina en segunda intención, consiguieron micción espontánea. Finalmente, el 50\% (7/14) de los pacientes portadores de catéter tras el tratamiento con $4 \mathrm{mg}$ de doxazosina consiguieron micción con $8 \mathrm{mg}$. En el análisis de regresión logistica, ninguna de las variables analizadas permitió predecir la respuesta al tratamiento.

CONCLUSIÓN: El tratamiento durante 7 días con $4 \mathrm{mg}$ de doxazosina de liberación sostenida permite retirar con mayor éxito el catéter tras RAO por HBP. Con este tratamiento, el $60 \%$ de los pacientes consiguen reanudar la micción espontánea. Incrementando la dosis a $8 \mathrm{mg}$ es posible la retirada del catéter hasta en la mitad de los pacientes inicialmente no respondedores. No es posible predecir, antes de la retirada del catéter, que pacientes conseguirán la micción espontánea.

PALABRAS CLAVE: Hiperplasia benigna de próstata. Retención aguda de orina. Doxazosina.

\section{ABSTRACT}

DOXAZOSIN IN THE GASTROINTESTINAL THERAPEUTIC SYSTEM (GITS) FORMULATION AND TRIAL WITHOUT CATHETER AFTER ACUTE URINARY RETENTION DUE TO BPH. DOSE INCREASE ACTION ON RECOVERY EFFECT

OBJECTIVE: Prospective and randomised study to assess the effectiveness of doxazosin in sustained release formulation in Acute Urinary Retention (AUR) treatment due to benign prostatic hyperplasia (BPH).

MATERIAL AND METHODS: The trial was carried out with a cohort of 40 males who had all suffered their first attack of AUR caused by BPH. Twenty were randomly selected and treated for 7 days with $4 \mathrm{mg}$ of sustained release doxazosin before removing the catheter. The patients not treated that could not spontaneously urinate were also administered $4 \mathrm{mg}$ of doxazosin. Finally, all the patients that still had a catheter due to unsuccessful removal were treated with $8 \mathrm{mg}$ of doxazosin and the percentage of patients responding to treatment was assessed. The predictive value of the response to treatment for age, IPSS, QoL, retained urine volume, prostate volume and the evolution time of the prostratism was determined by means of logistic regression analysis.

RESULTS: $82.5 \%$ of the patients (33/40) could urinate after removal of the catheter. $84.8 \%(28 / 33)$ were treated with doxazosin $(21$ with $4 \mathrm{mg}$ and 7 with $8 \mathrm{mg}$ ). In the first attempt at removal, $60 \%$ of the patients $(12 / 20)$ treated with $4 \mathrm{mg}$ of doxazosin could spontaneously urinate, while only $25 \%(5 / 20)$ of those not treated, $p=0.02$. Similarly, $60 \%$ of the patients $(9 / 15)$ treated with $4 \mathrm{mg}$ of doxazo$\sin$ in the second attempt could spontaneously urinate. Fifty per cent (7/14) of the patients still with a catheter, after the treatment with $4 \mathrm{mg}$ of doxazosin, could urinate with $8 \mathrm{mg}$. In the logistic regression analysis, none of the variables analyzed allowed us to predict the response to the treatment.

CONCLUSION: The treatment for 7 days with $4 \mathrm{mg}$ of sustained release doxazosin shows greater success when removing the catheter after suffering AUR due to BPH. With this treatment, $60 \%$ of the patients could spontaneously urinate again. By increasing the dose to $8 \mathrm{mg}$, the catheter can be removed in half the patients that did not initially respond. Before removing the catheter it is not possible to predict which patients would be able to spontaneously urinate.

KEY WORDS: Benign prostatic hyperplasia. Acute urinary retention. Doxazosin. 
$\mathrm{L}^{\mathrm{a}}$ a hiperplasia benigna de próstata (HBP) es una condición frecuente relacionada con la edad y es la principal causa en varones de sintomas crónicos del tracto urinario bajo. La retención aguda de orina (RAO) es una importante complicación de la HBP que se presenta en alrededor del $10 \%$ de los pacientes ${ }^{1}$. Ha sido estimado que la RAO es la indicación de la cirugía en el 25 a 30\% de los pacientes sometidos a prostatectomía por $\mathrm{HBP}^{2}$. Diversos estudios demuestran que los pacientes que se someten a la cirugía siendo portadores de una sonda urinaria tienen un riesgo mayor de complicaciones operatorias y post-operatorias, transfusiones y muerte. La sonda uretral conlleva, además de un detrimento de la calidad de vida, un índice de colonización bacteriana superior al $4 \%$ por día ${ }^{3}$. Por lo tanto, es frecuente intentar la retirada del catéter tras un episodio de RAO obteniéndose micciones espontáneas en el 20-30\% de los pacientes ${ }^{4}$.

Está demostrado que el bloqueo de los receptores $\alpha 1$ adrenérgicos mejoran los síntomas crónicos del tracto urinario bajo debidos a HBP reduciendo la resistencia uretral al flujo de vaciado vesical. Se conoce que el incremento de la resistencia al flujo juega un papel importante en la RAO por HBP. El efecto urodinámico del bloqueo de estos receptores probablemente reduzca la frecuencia de RAO y los requerimientos de cirugía en estos pacientes. Su empleo previo a la retirada del catéter está fisiológicamente justificado. El mecanismo de acción no parece estar relacionado con la selectividad por los receptores en el estroma prostático motivo por el que han sido comunicados resultados favorables con los distintos bloqueantes $\alpha 1$ adrenérgicos ${ }^{5-7}$. El agente ideal para ser empleado antes de la retirada del catéter debe ser bien tolerado, con una rápida acción, larga vida media y sin fraccionamiento de dosis. La doxazosina, en una formulación de liberación retardada, ha demostrado mejorar los síntomas de vaciado debidos a HBP incrementando el flujo urinario, con un mínimo efecto sobre la presión arterial ${ }^{8}$. El paciente puede iniciar el tratamiento a dosis terapéuticas sin necesidad de fraccionamiento de dosis. Los estudios clínicos demuestran una mejoría flujométrica y sintomática a la semana del inicio del tratamiento ${ }^{9}$.
En este estudio prospectivo y randomizado se pretende evaluar el efecto de la doxazosina en formulación de liberación retardada en la retirada del catéter tras RAO en una cohorte de pacientes con HBP.

\section{MATERIAL Y MÉTODOS}

\section{Pacientes $y$ diseño}

Se estudia una cohorte de 40 varones consecutivos con historia previa de sintomas del tracto urinario inferior debidos a HBP y que han sufrido un primer episodio de RAO. Todos son portadores de catéter uretral en el momento del estudio. Se recogen los datos de la edad, IPSS, volumen de orina retenido, volumen prostático determinado mediante ecografía suprapúbica y tiempo de evolución de los síntomas del tracto urinario inferior debidos a HBP. Veinte pacientes son tratados aleatoriamente, durante 7 días, con $4 \mathrm{mg}$ de doxazosina de liberación retardada con posterior retirada del catéter y comprobación de micciones espontáneas. Los otros 20 pacientes no tratados son portadores también de catéter durante 7 días. De ellos, los que no restablecieron las micciones espontáneas recibieron también $4 \mathrm{mg}$ de doxazosina, tras lo que se realiza otro intento de retirada. Finalmente, todos los pacientes que persistieron con catéter por retirada infructuosa fueron tratados con $8 \mathrm{mg}$ de doxazosina, durante 7 días más, evaluándose el porcentaje de pacientes respondedores.

\section{Análisis estadístico}

Se analizan la distribución de las variables clínicas entre los grupos tras la randomización y en función de la respuesta al tratamiento con tablas de contingencia (Chi-cuadrado). Se determina mediante análisis de regresión logística el valor predictivo de la respuesta al tratamiento de las variables clínicas recogidas. Se ha empleado el programa estadístico SPSS 6.0 ${ }^{10}$.

\section{RESULTADOS}

De los 40 pacientes incluidos en este estudio, tras la randomización 20 fueron tratados durante 7 días con $4 \mathrm{mg}$ de doxazosina de liberación retardada. Las características clínicas de los pacientes en esta primera fase del estudio se describen en la Tabla I, donde se observa que las 
TABLA I

VARIABLES CLÍNICAS DE LOS PACIENTES ALEATORIZADOS EN LA PRIMERA FASE DEL ESTUDIO

\begin{tabular}{|l|c|c|}
\hline & Sin tratamiento & Dox 4 \\
\hline $\mathrm{N}$ & 20 & 20 \\
\hline Edad & $71,4(68,0 ; 74,7)$ & $73,8(69,4 ; 78,2)$ \\
$<65$ & 5 & 5 \\
$65-75$ & 5 & 10 \\
$>75$ & 10 & 5 \\
\hline IPSS & $17,3(15,7 ; 18,9)$ & $16,1(14,3 ; 17,9)$ \\
\hline QoL & $3,8(3,6 ; 4,1)$ & $4,1(3,8 ; 4,4)$ \\
\hline Vol. Prostático & $51,7(44,5 ; 58,8)$ & $53,7(44,7 ; 62,7)$ \\
$<30$ & 2 & 1 \\
$30-50$ & 9 & 9 \\
$>50$ & 9 & 10 \\
\hline Vol. RAO & $932(830,1030)$ & $1000(914,1086)$ \\
\hline Síntomas & $39,6(30,2 ; 48,9)$ & $37,8(30,5 ; 45,1)$ \\
\hline
\end{tabular}

Vol. prostático: cc.

Vol. RAO: cc.

Síntomas: meses

variables fueron comparables entre los dos grupos. Tras la retirada del catéter, 17 pacientes reanudaron micciones espontáneas (5 pacientes no tratados y 12 tratados, $\mathrm{p}=0,02$ ). La odds ratio a favor de los pacientes tratados fue 4,5 (95\% IC $1,2 ; 16,5)$. No se encontraron diferencias en las variables clínicas analizadas entre los pacientes que reanudaron las micciones en esta primera fase del estudio (Tabla IIa). Los 15 pacientes no tratados y portadores de sonda por nueva RAO, fueron tratados en un segundo intento durante 7 días con $4 \mathrm{mg}$ de doxazosina de liberación retardada antes de la nueva retirada. Nueve pacientes (60\%) consiguieron micción espontánea, el mismo porcentaje de respuesta que en la primera fase. Finalmente, los 14 pacientes portadores de catéter tras el tratamiento con $4 \mathrm{mg}$ de doxazosina ( 8 pacientes de la primera fase y 6 de la segunda) fueron tratados durante 7 días con 8 $\mathrm{mg}$ de doxazosina. Siete pacientes (50\%) reanudaron micciones espontáneas tras la retirada de la sonda (Figura 1). En total, el $82,5 \%$ de los pacientes (33/40) consiguieron la micción tras la retirada del catéter. El 84,8\% (28/33) fueron tratados con doxazosina ( 21 con $4 \mathrm{mg}$ y 7 con $8 \mathrm{mg}$ ). Al final del estudio no se encontraron diferencias, en la mayoría de las variables clínicas analizadas, entre los pacientes que reanudaron las micciones

\section{TABLA IIA}

VARIABLES CLÍNICAS EN FUNCIÓN

DE LA REANUDACIÓN DE LAS MICCIONES TRAS LA RETIRADA DEL CATÉTER: (A) EN LA PRIMERA FASE $\mathrm{Y}(\mathrm{B})$ AL FINAL DEL ESTUDIO

\begin{tabular}{|c|c|c|}
\hline & $\oplus$ & $\varnothing$ \\
\hline $\mathrm{N}$ & 17 & 23 \\
\hline $\begin{array}{l}\text { Edad } \\
<65 \\
65-75 \\
>75\end{array}$ & $\begin{array}{c}72,8(68,7 ; 77,0) \\
4 \\
5 \\
8\end{array}$ & $\begin{array}{c}72,3(68,6 ; 76,0) \\
6 \\
10 \\
7\end{array}$ \\
\hline IPSS & $16,2(14,7 ; 17,7)$ & $17,0(15,3 ; 18,8)$ \\
\hline GoL & $4,0(3,7 ; 4,4)$ & $3,9(3,7 ; 4,2)$ \\
\hline $\begin{array}{l}\text { Vol. Prostático } \\
<30 \\
30-50 \\
>50\end{array}$ & $\begin{array}{c}47,9(38,5 ; 57,3) \\
2 \\
11 \\
4\end{array}$ & $\begin{array}{c}56,2(49,4 ; 63,1) \\
1 \\
8 \\
14\end{array}$ \\
\hline Vol. RAO & $976(870,1082)$ & $958(872,1045)$ \\
\hline Sintomas & $38,2(27,1 ; 49,1)$ & $39,1(32,4 ; 45,6)$ \\
\hline $\begin{array}{l}\text { Tratamiento } \\
\text { Sin tto. } \\
\text { Dox } 4\end{array}$ & $\begin{array}{c}5(25 \%) \\
12(60 \%)\end{array}$ & $\begin{array}{c}15(75 \%) \\
8(40 \%)\end{array}$ \\
\hline
\end{tabular}

(Tabla IIb). Tan sólo se encontraron diferencias en el IPSS, observándose una puntuación menor en los pacientes que consiguieron micción tras la retirada del catéter, 16,3 vs. 18,$7 ; \mathrm{p}<0,05$. Sin embargo, en el análisis de regresión logística, ninguna de las variables analizadas permitió predecir la respuesta al tratamiento (Tabla III).

\section{DISCUSIÓN}

La RAO es una importante complicación de la HBP que se presenta en alrededor del $10 \%$ de los pacientes ${ }^{1}$. Ha sido demostrado que los pacientes que se someten a la cirugía siendo portadores de una sonda urinaria tienen un riesgo mayor de complicaciones. Por este motivo es frecuente intentar la retirada del catéter previa a la prostatectomía. Diversos estudios han demostrado la utilidad del tratamiento con un bloqueante de los receptores $\alpha 1$ adrenérgicos con esta indicación ${ }^{5-}$ 7. En este estudio los pacientes que son tratados con $4 \mathrm{mg}$ de doxazosina de liberación retardada 7 días antes de la retirada del catéter tras RAO por HBP, tienen 4,5 veces más posibilidades de res- 


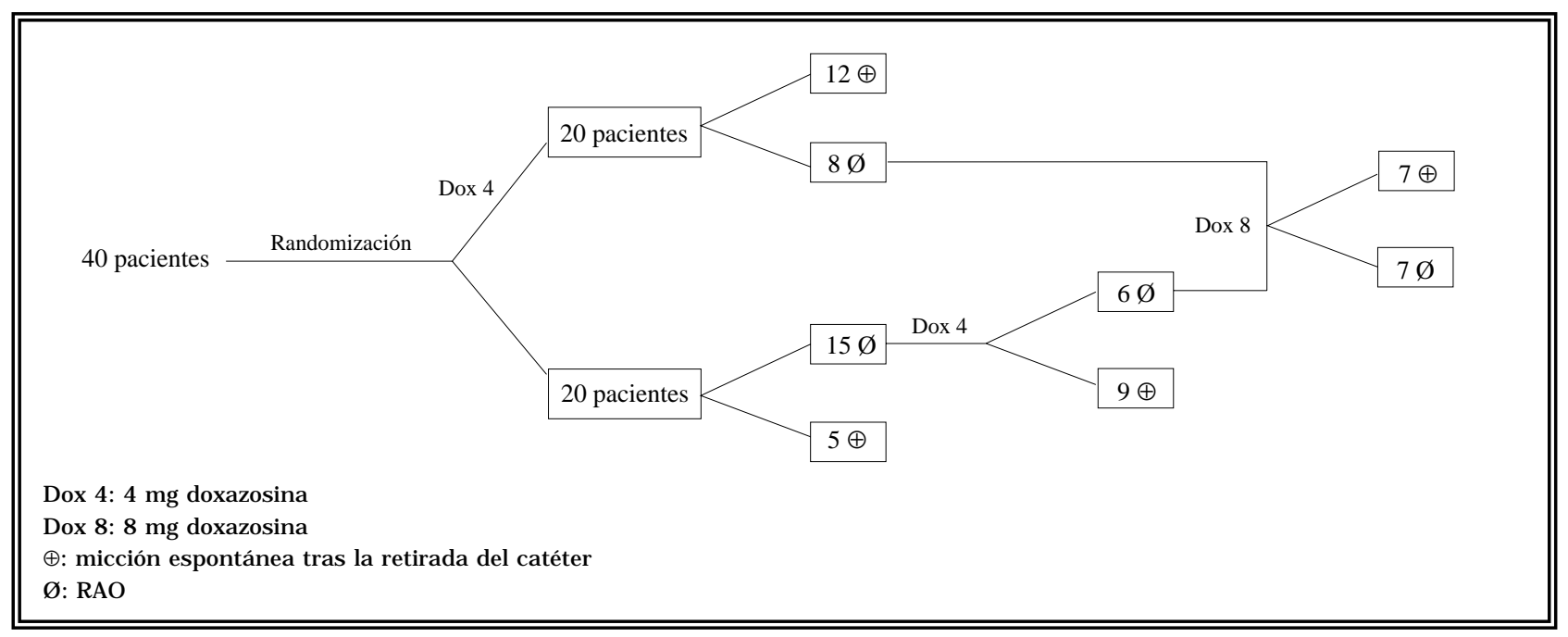

FIGURA 1. Esquema del estudio y resultados tras la retirada del catéter.

TABLA IIB

VARIABLES CLÍNICAS EN FUNCIÓN DE LAREANUDACIÓN DE LAS MICCIONES TRAS LA RETIRADA DEL CATÉTER: (A) EN LA PRIMERA FASE Y (B) AL FINAL DEL ESTUDIO

\begin{tabular}{|c|c|c|}
\hline & $\oplus$ & $\varnothing$ \\
\hline $\mathrm{N}$ & 33 & 7 \\
\hline $\begin{array}{l}\text { Edad } \\
<65 \\
65-75 \\
>75\end{array}$ & $\begin{array}{c}72,4(69,4 ; 75,4) \\
10 \\
9 \\
14\end{array}$ & $\begin{array}{c}73,4(65,8 ; 81,0) \\
0 \\
6 \\
1\end{array}$ \\
\hline IPSS* & $16,3(14,9 ; 17,6)$ & $18,7(17,0 ; 20,3)$ \\
\hline GoL & $4,0(3,8 ; 4,2)$ & $3,8(3,2 ; 4,5)$ \\
\hline $\begin{array}{l}\text { Vol. Prostático } \\
<30 \\
30-50 \\
>50\end{array}$ & $\begin{array}{c}51,9(45,4 ; 58,5) \\
3 \\
17 \\
13\end{array}$ & $\begin{array}{c}56,4(48,1 ; 64,7) \\
0 \\
2 \\
5\end{array}$ \\
\hline Vol. RAO & $948(885,1011)$ & $1050(789,1310)$ \\
\hline Síntomas & $38,2(31,4 ; 45,0)$ & $41,1(32,4 ; 49,2)$ \\
\hline $\begin{array}{l}\text { Tratamiento } \\
\text { Sin tto } \\
\text { Dox } 4 \\
\text { Dox } 8\end{array}$ & $\begin{array}{c}5(25 \%) \\
21(60 \%) \\
7(50 \%)\end{array}$ & $\begin{array}{c}0(0 \%) \\
0(0 \%) \\
7(50 \%)\end{array}$ \\
\hline
\end{tabular}

${ }^{*} \mathrm{p}<0,05$

tablecer las micciones espontáneas que los pacientes no tratados. Igualmente, el porcentaje de restablecimiento de las micciones espontáneas se mantiene entre los pacientes tratados por segunda intención. Sin embargo, tan sólo el 25\% de los pacientes no tratados consiguen micción. Este porcentaje es similar a los comunicados previamente en los estudios no controlados observacionales y en los controlados con placebo ${ }^{4-6,11}$. No obstante, no existe suficiente experiencia sobre el tipo de $\alpha$ antagonista y tiempo óptimo de tratamiento antes de la retirada del catéter. McNeill et al. ${ }^{6}$ randomizaron 81 pacientes con RAO tratando 40 con $5 \mathrm{mg}$ de alfuzosina dos veces al día y 41 con placebo durante 48 horas. Observaron una mayor probabilidad de micción espontánea al retirar a las 24 horas el catéter en los pacientes tratados con alfuzosina ( $55 \%$ vs $29 \%)$. Igualmente, Kim et al. ${ }^{7}$ consiguieron una retirada exitosa del catéter en el $88 \%$ de los 33 pacientes

\section{TABLA III}

ANÁLISIS DE REGRESIÓN LOGÍSTICA EN LA PREDICCIÓN DE LA RESPUESTA AL TRATAMIENTO

\begin{tabular}{|l|c|c|c|}
\hline & B & Exp B & $95 \%$ IC Exp B \\
\hline Constante & $-7,866$ & & \\
\hline Edad & $-0,033$ & 0,9967 & 0,$8846 ; 1,1231$ \\
\hline IPSS & 0,3524 & 1,4224 & 0,$911 ; 2,2208$ \\
\hline GoL & $-1,0617$ & 0,3459 & 0,$509 ; 2,3503$ \\
\hline Vol. Prostático & 0,029 & 1,0293 & 0,$9609 ; 1,103$ \\
\hline Vol. RAO & 0,0024 & 1,0024 & 0,$9977 ; 1,0071$ \\
\hline Síntomas & 0,1562 & 1,1691 & 0,$5405 ; 2,5285$ \\
\hline
\end{tabular}


tratados con $0,4 \mathrm{mg}$ de tamsulosina durante una media de 6,8 días. No está claro que un tratamiento más prolongado antes de la retirada del catéter produzca un mayor porcentaje de éxito. Aunque el efecto urodinámico del bloqueo $\alpha$ se produce a las pocas horas de su administración parece que el máximo incremento en el flujo y el máximo beneficio sintomático se obtiene a las semanas del inicio del tratamiento. Se ha sugerido que el incremento del tono simpático a nivel de la próstata y el cuello vesical puede afectar a la actividad del músculo detrusor por lo que el mantenimiento del catéter unos días tras el inicio del bloqueo $\alpha$ puede ser beneficioso. La duración del sondaje ha sido comunicado como un factor importante en la reanudación de la micción en los pacientes randomizados para su mantenimiento por diferente espacio de tiempo. Se ha comunicado el restablecimiento de las micciones en el $44 \%$ de los pacientes tras la retirada inmediata del catéter, $51 \%$ tras 2 días y $62 \%$ tras 7 días ${ }^{12}$.

No hemos encontrado en la literatura ningún trabajo que analice la dosis como un factor para el restablecimiento de la micción. En la experiencia de Kim et al. ${ }^{7}$ tres pacientes quedaron en RAO tras un intento inicial de retirada reanudando las micciones todos ellos tras 4 días más de tamsulosina. En nuestro estudio e incrementando las dosis hasta $8 \mathrm{mg}$ de doxazosina el $50 \%$ de los pacientes inicialmente no respondedores a $4 \mathrm{mg}$ consiguieron micciones espontáneas. Hacen falta más estudios para atribuir el incremento de pacientes respondedores al aumento de la dosis o al mantenimiento durante más días del catéter.

La edad, la puntuación GoL del IPSS, el volumen retenido y el volumen prostático han sido correlacionados con la evolución tras la retirada del catéter ${ }^{6,7,12,13}$. Para McNeill et al. ${ }^{6}$ la edad es un importante factor que influye en el restablecimiento de la micción, reduciéndose la probabilidad del éxito. Ningún paciente de más de 85 años consiguió la retirada del catéter. Probablemente este dato expresa cronológicamente la historia natural de los síntomas de prostatismo $u$ otros factores predictivos como el residuo post-miccional. Para estos autores, el volumen de orina retenido y el volumen prostático no tienen valor pronóstico. Ha sido comunicado en estudios obser- vacionales que el volumen retenido se relaciona con la reanudación de la micción espontánea y el incremento en el volumen prostático es un reconocido factor de riesgo para el desarrollo de la RAO. Djavan et al. ${ }^{12}$ encuentran que un volumen inferior a $1000 \mathrm{ml}$ se asocia con un mayor éxito, mientras que se recomienda un cateterismo más prolongado si el volumen es superior a $1300 \mathrm{ml}$. En relación con el tamaño prostático, aunque un volumen superior a $30 \mathrm{ml}$ se ha relacionado con un riesgo 3 veces incrementado de $\mathrm{RAO}^{13}$, ninguno de los estudios analizados lo relaciona positivamente con la reanudación de las micciones. Para Kim et al. ${ }^{7}$ una elevada puntuación en el GoL del IPSS se relaciona con el fallo de la respuesta a la tamsulosina y predice la necesidad de RTU a corto plazo. En el análisis final de las variables clínicas en los pacientes que consiguieron micción espontánea encontramos diferencias estadísticamente significativas tan sólo en la puntuación del IPSS (16,3 vs. 18,7 ; $p<0,05)$. Sin embargo, en el análisis de regresión logística ninguno de los factores mencionados se relacionó con la reanudación de las micciones, por lo que inicialmente todo paciente puede ser tributario de tratamiento previo a la retirada del catéter.

En conclusión, este estudio demuestra que el tratamiento durante 7 días con $4 \mathrm{mg}$ de doxazosina de liberación retardada es útil para incrementar el porcentaje de micciones espontáneas tras la retirada del catéter. Probablemente, los pacientes no respondedores pueden beneficiarse de un mayor tiempo de sondaje o de un incremento de la dosis. Esto puede beneficiar a los pacientes en espera de cirugía prostática reduciendo la morbilidad perioperatoria y la calidad de vida. Hacen falta estudios evolutivos para determinar si el mantenimiento del tratamiento permite reducir nuevos episodios de RAO y la necesidad de cirugía por HBP.

\section{REFERENCIAS}

1. WASSON JH, REDA DJ, BRUSKEWITZ RC, ELINSON J, KELLER AM, HENDERSON WG.: A comparison of transurethral surgery with watchful waiting for moderate symptoms of benign prostatic hyperplasia. The veterans affairs cooperative study group on transurethral resection of the prostate. $N$ Engl $J$ Med 1995; 332: 75-79. 
2. MEBUST WK, HOLTGREWE HL, COCKETT ATK, PETERS PC and the Writing Committee.: Transurethral prostatectomy: immediate and postoperative complications. A co-operative study of 12 participating institutions evaluating 3885 patients. $J$ Urol 1989; 141: 243-247.

3. GARIBALDI RA, BURKE JP, DICKMAN ML, SMITH CB.: Factors predisposing to bacteriuria during indwelling urethral catheterisation. N Engl J Med 1974; 291: 215-219.

4. TAUBE M, GAJRAJ H.: Trial without catheter following acute retention of urine. $\mathrm{Br} J$ Urol 1989; 63: 180-182.

5. PEREPANOVA TS, KAMALOV AA, SINIUKHIN VN, GOROKHNOV AV, KHAZAN PL, ORLOVA EV.: Doksazozin "Cardura" in acute urinary retention caused by benign prostatic hyperplasia. Urologia 2001; 3: 18-20.

6. MCNEILL SA, DARUWALA PD, MITCHELL IDC, SHEARER MG, HARGREAVE TB.: Sustained-release alfuzosin and trial without catheter after acute urinary retention: a prospective, placebo-controlled trial. BJU Int 1999; 84: 622-627.

7. KIM HL, KIM JC, BENSON DA, BALES GT, GERBER GS.: Results of treatment with tamsulosin in men with acute urinary retention. Tech Urol 2001; 7 (4): 256-260.

8. CHUNG M, VASHI V, PUENTE J, SWEENEY M, MEREDITH P.: Clinical pharmacokinetics of doxazosin in a controlled-release gastrointestinal therapeutic system (GITS) formulation. Br J Clin Pharmacol 1999; 48: 678-687.
9. ANDERSEN M, DAHLSTRAND C, HOYE K.: Double-blind trial of the efficacy and tolerability of doxazosin GITS, doxazosin standard and placebo in patients with BPH. Eur Urol 2000; 38: 400-409.

10. SPSS for Windows. Base system user's guide release 6.0. Chicago. SPSS inc. 1993.

11. MURRAY K, MASSCY A, FENELEY RCL.: Acute urinary retention- a urodynamic assessment. Br J Urol 1984; 56: 468-473.

12. DJAVAN B, SHARIAT S, OMAR M, ROEHRBORN CG, MARBERGER M.: Does prolonged catheter drainage improve the chance of recovering voluntary voiding after urinary retention (AUR). Eur Urol 1998; 33 (suppl): 1.

13. JACOBSEN SJ, JACOBSON DJ, GIRMAN CJ, ROBERTS RO, RHODES T, GUESS HA, LIEBER MM.: Natural history of prostatism: risk factors for acute urinary retention. J Urol 1997; 158: 481-487.

Dr. J.A. Lorente Garín

Servicio de Urología.

Hospital del Mar

Passeig Maritim, 25-29

08003 Barcelona

(Trabajo recibido el 12 febrero de 2003) 